\title{
Capacitação de professores de escolas públicas de Tucuruí-PA na inserção de temas transversais
}

\author{
Training of public school teachers from Tucuruí-PA in the \\ insertion of cross-cutting themes
}

\author{
Daniele Lima dos Anjos Reis ${ }^{1,2}$, João Augusto de Souza Miranda ${ }^{3}$, \\ Thaísa Évelin Trevizan Reis', Edinelma Silva de Carvalho Mota ${ }^{1,4}$, Kátia Simone Kietzer2,5 \\ ' Universidade do Estado do Pará, Tucuruí (PA), Brasil. \\ ${ }^{2}$ Programa de Pós-graduação em Saúde da Amazônia, Centro de Ciência Biológicas e da Saúde, Universidade \\ do Estado do Pará, Belém (PA), Brasil. \\ ${ }^{3}$ Faculdade Carajás, Marabá (PA), Brasil. \\ 43ํㅡㄹ Centro Regional de Saúde, Secretaria de Estado de Saúde Pública do Pará, Castanhal (PA), Brasil. \\ ${ }^{5}$ Programa de Pós-graduação em Cirurgia e Pesquisa Experimental, Centro de Ciência Biológicas e da Saúde, \\ Universidade do Estado do Pará, Belém (PA), Brasil.
}

Recebido: Ago. 15, 2017 Aceito: Dez. 14, 2017

\section{COMO CITAR ESTE ARTIGO}

Reis DLA, Miranda JAS, Reis TET, Mota ESC, Kietzer KS. Capacitação de professores de escolas públicas de Tucuruí-PA na inserção de temas transversais. Interdisciplinary Journal of Health Education. 2017 Jul-Dez;2(2):119-126. https://doi. org/10.4322/ijhe.2017.006

\section{CORRESPONDÊNCIA}

Daniele Lima dos Anjos Reis Universidade do Estado do Pará Rua Tocantins, 55, Vila Tropical, CEP 68455-761, Tucuruí (PA), Brasil anjo.daniele@hotmail.com

\section{FONTE DE FINANCIAMENTO}

Pró-reitoria de Extensão (PROEX) da Universidade do Estado do Pará, pelo Programa Campus Avançado, em 2010.

\section{CONFLITO DE INTERESSE}

Os autores declararam não

haver conflitos de interesse.

O estudo foi realizado na Universidade do Estado Pará, Tucuruí (PA), Brasil.

Este trabalho foi apresentado no 14ํㅡㄹ CBCENF - Congresso Brasileiro dos Conselhos de Enfermagem, em 2011.

Todos os autores leram e aprovam a versão final submetida ao Interdisciplinary Journal of Health Education (IJHE).

\section{RESUMO}

Contextualização: A inclusão de temas transversais torna-se cada vez mais frequente nos currículos escolares nacionais. As escolas apresentam dificuldades em estabelecer debates acerca desses assuntos, contribuindo para o aumento negativo dos indicadores de saúde. Assim, destaca-se a educação continuada em saúde como estratégia de capacitação profissional. Objetivou-se contribuir para a capacitação de professores de $5^{\text {a }}$ a $8^{\text {a }}$ séries de escolas públicas do município de Tucuruí-PA como agentes multiplicadores de temas transversais como Sexualidade, Orientação Sexual, Métodos Contraceptivos, Gravidez na Adolescência, Abuso Sexual, Aborto, DSTs, HIV/AIDS, Uso de Drogas/Violência e Promoção à Saúde. Descrição da Experiência: Participaram 20 professores liberados pela Secretaria de Educação. As atividades teórico-práticas foram coordenadas por 01 docente da Universidade do Estado do Pará (UEPA), e executadas no período de 13 a 17 de setembro de 2010, por 02 acadêmicos de Enfermagem e 01 acadêmica de Educação Física, com carga horária total de 40 horas e certificação final. Os temas transversais foram tratados por meio de aulas expositivas e interativas, dinâmicas de grupo e atividades práticas; nos turnos manhã e tarde, nas dependências físicas da UEPA, núcleo de Tucuruí. Resultados e Impactos: Este projeto propiciou a apresentação de ferramentas metodológicas para a abordagem integrada dos temas transversais às disciplinas escolares pelos participantes e contribuiu para a qualificação da formação continuada de professores da educação básica, por meio da articulação Universidade-Escola. Apesar de algumas dificuldades encontradas durante a execução e pela importância dos temas tratados, os autores do trabalho foram convidados a estender tais ações para eventos em outras instituições e municípios. Considerações Finais: Evidenciou-se que é necessário que as relações entre comunidade acadêmica e escolar sejam fomentadas por meio da implementação de projetos de pesquisa e de extensão que estimulem o planejamento e desenvolvimento de ações para a promoção da saúde de forma permanente nas escolas.

PALAVRAS-Chave: Educação. Saúde. Capacitação de professores.

\section{ABSTRACT}

Contextualization: The inclusion of cross-cutting themes is becoming more frequent in national school curricula. Studies show that schools present difficulties in establishing debates on these subjects, which contributes to the negative increase of health indicators. Thus, continuing education in health is highlighted as a strategy for professional training. The objective was to contribute to the training of teachers from 5 th to 8 th grade public schools in the city of Tucuruí-PA as multipliers of cross-cutting themes such as Sexuality, Sexual Orientation, Contraceptive Methods, Adolescence Pregnancy, Sexual Abuse, Abortion, STDs, HIV/AIDS, Drug Use/Violence and Health Promotion. Description of the Experience: Twenty teachers released by the Department of Education participated. The theoretical-practical activities were coordinated by $\mathrm{O} 1$ lecturer from the University of the State of Pará (UEPA), and executed in the period from September 13 to 17, 2010, according to a timetable elaborated by 02 nursing students and $\mathrm{O} 1$ academic of Physical Education, with Total workload of 40 hours and final certification. The cross-cutting themes were treated through lectures with effective interaction of educators, group dynamics and varied practical activities; In the morning and afternoon, in the physical dependencies of UEPA, core of Tucuruí. Results and Impacts: This project provided the presentation of methodological tools for the integrated approach of the subjects transversal to the school disciplines by the participants and contributed to the qualification of 
the continuing education of primary education teachers through the University-School articulation. Despite some difficulties encountered during the execution and the importance of the topics discussed, the authors of the work were invited to extend such actions to events in other institutions and municipalities. Final Considerations: It was evidenced that it is necessary that the relations between academic and school community be fomented through the implementation of research and extension projects that stimulate the planning and development of actions for the promotion of health of permanent form in the schools.

KEYWORDS: Education. Health. Teacher training.

\section{Contextualização}

Os Temas Transversais são conceituados como conteúdos que estão voltados para a realidade social, correspondendo a questões importantes, urgentes e presentes no cotidiano ${ }^{1}$. De acordo com os Parâmetros Curriculares Nacionais (PCNs), o conjunto de Temas Transversais a serem abordados são: Ética, Meio Ambiente, Pluralidade Cultural, Saúde, Orientação Sexual e Trabalho e Consumo, de forma que os temas podem ser priorizados e contextualizados de acordo com as diferentes realidades locais e regionais ${ }^{2}$.

A inclusão de tais temas torna-se cada vez mais frequente nos currículos escolares nacionais, cuja atualização é expressa nos PCNs. Essa atualização curricular defende a inserção interdisciplinar de Temas Transversais no trabalho educativo das escolas e dos educadores, o que recebeu o nome de transversalidade $\mathrm{e}^{1,3}$.

Assim, o professor tem papel fundamental na abordagem desses conteúdos inseridos no cotidiano dos alunos e, paradoxalmente, as escolas apresentam dificuldades em estabelecer debates acerca desses assuntos, contribuindo para o aumento negativo dos indicadores de saúde e desviando-se da sua função de preparar o aluno para a cidadania .

A educação continuada em saúde, nesse panorama, destaca-se como uma estratégia para capacitar profissionais para planejar, desenvolver, avaliar e reestruturar os serviços aos quais pertencem, considerando o contexto familiar, comunitário e social do ser humano ${ }^{5}$. Falando em docência, formação continuada são todas as ações de formação realizadas pelo professor, como as pós-graduações e os cursos oferecidos por universidades, secretarias de ensino e pelo próprio ambiente escolar ${ }^{6}$.

A execução deste projeto justificou-se pela necessidade de fornecer ferramentas que pudessem auxiliar professores no desenvolvimento de atividades capazes de integrar temas que devem ser tratados nas escolas, principalmente devido à alta vulnerabilidade dos jovens e por envolverem problemáticas sociais atuais. Considerando a realidade local, objetivou-se contribuir para a capacitação de professores de $5^{\mathrm{a}}$ a $8^{\mathrm{a}}$ séries de escolas públicas do município de Tucuruí-PA, como agentes multiplicadores dos temas transversais Sexualidade, Orientação Sexual, Métodos Contraceptivos, Gravidez na Adolescência, Abuso Sexual, Aborto, DSTs/HIV/AIDS, Uso de Drogas/Violência e Promoção à Saúde.

\section{Descrição da experiência}

Este projeto de extensão fez parte do Programa Campus Avançado da Universidade do Estado do Pará (UEPA), em 2010. Participaram 20 professores de $5^{\text {a }}$ a $8^{\mathrm{a}}$ séries de escolas variadas de Tucuruí-PA, selecionados e liberados pela Secretaria de Educação do município citado.

As atividades teórico-práticas propostas foram realizadas nas dependências físicas da UEPA, núcleo de Tucuruí-PA, coordenadas por 01 docente desta instituição e executadas no período de 13 a 17 de setembro de 2010, com cronograma prévio, por 02 acadêmicos de enfermagem e 01 acadêmica de educação física. A carga horária total foi de 40 horas e todos os participantes receberem certificados emitidos pela UEPA. As ações foram realizadas por meio de variadas estratégias metodológicas, 
como aulas interativas com auxílio de data-show, dinâmicas de grupos e atividades práticas para o favorecimento das situações de ensino-aprendizagem dos professores, conforme descrito a seguir.

Dia: 13/09/2010

MANHÃ-Sexualidade na Adolescência. Aula Expositiva-interativa: foi realizada abordagem geral sobre sexualidade na adolescência, fatores sócio-econômico-culturais que permeiam este evento, alterações anátomo-fisiológicas nos adolescentes, sistema reprodutor masculino e feminino e mitos/tabus sobre a sexualidade. Dinâmica de Grupo - Dicionário Sexual dos Adolescentes: objetivou-se familiarizar os educadores com termos voltados à sexualidade/relação sexual utilizados pelos adolescentes. A dinâmica consistiu em separar a turma em 04 grupos, os quais construíram os Dicionários Sexuais dos Adolescentes, e ao término de 20 minutos expuseram para a turma os termos identificados em suas rotinas. A cada grupo foi entregue 01 folha A4 e 01 caneta. Atividade Prática - Estudo dirigido: a turma foi dividida em 05 grupos, sendo que cada um deles recebeu um texto relacionado ao conteúdo trabalhado, entre eles "Orientação Sexual na Família e na Escola", "Virgindade", "O Sexo na Adolescência", "O Sexo e as Religiões" e "A Igreja e a Proibição do Uso de Camisinha". Foi dado um tempo de 25 minutos para a leitura e reflexão dos textos pelos grupos para após ser realizada a exposição para a turma e debate. Ressalta-se que os informativos foram extraídos da internet, não havendo preocupação com as fontes das informações, com o intuito de causar discussão sobre a veracidade das informações abordadas, bem como, sobre o modo que as informações chegam aos adolescentes através do mundo virtual, o qual muitas vezes disponibiliza conteúdos deturpados e com poder e banalização da sexualidade. Enfatizou-se a relevância da articulação das diversas áreas do saber para discutir sobre sexualidade na sala de aula, assim "[...] cada professor poderia, para começar, tentar mostrar que os conteúdos que ensina em suas aulas não estão isolados e se relacionam de algum modo com tudo o que o aluno aprende na escola"7 (p. 37).

TARDE - Respeito à Sexualidade. Aula Expositiva-interativa: constou de um histórico, conceito, fases de desenvolvimento da sexualidade e transtornos de identidade de gênero. Foram citadas metodologias e atividades que podem ser desenvolvidas com os alunos. Dinâmica de Grupo - Vantagens e desvantagens de ser homem e mulher: a sala foi dividida em 04 grupos, onde 02 deveriam listar os lados bom e ruim de ser mulher e os outros 02 os lados bom e ruim de ser homem. Em seguida, um representante de cada grupo leu as listas, promovendo assim uma discussão sobre as diferenças entre homem e mulher, enfatizando o respeito que deve haver ao gênero. Atividade Prática - Oficina: consistiu na montagem de painéis envolvendo o tema da sexualidade. Foram formados 04 grupos e solicitado que organizassem os painéis utilizando imagens que manifestassem a sexualidade direta ou indiretamente, para posterior socialização. Entregamos para cada grupo papel $40 \mathrm{~kg}$, papel cartão, revistas, tesoura, cola e pincel atômico.

\section{Dia: $14 / 09 / 2010$}

MANHÃ - Adolescência e Métodos Contraceptivos. Aula Expositiva-interativa: explanou-se sobre a importância da educação sexual na adolescência, mostrando dados que comprovam a necessidade de se iniciar essa abordagem nesta fase. Dinâmica de Grupo - As cores da prevenção: sua realização durou em média 15 minutos. Foi fixado na parede um papel $40 \mathrm{Kg}$ com os 04 grupos de métodos contraceptivos apresentados na aula expositiva (Métodos de Barreira, Comportamentais, Hormonais e Definitivos). A seguir, foi solicitado aos participantes que listassem no papel fixado os métodos conhecidos, de acordo com o grupo ao qual ele pertencia e com a cor do pincel atômico adequado (explicado previamente aos professores) ao uso na adolescência. Atividade Prática - Oficina: Tendo em vista que a intersetorialidade entre saúde e educação deve 
fazer parte do cotidiano escolar, foi sugerida essa atividade em que os participantes deveriam organizar uma Feira Educacional na sua escola, com o foco na disseminação de conhecimento sobre os métodos contraceptivos. Solicitamos a formação de 04 grupos, sendo que cada um deveria planejar: o tema da Feira Educacional; o cronograma das atividades; os métodos utilizados para a realização do evento; os resultados esperados com o evento. Foram distribuídos para cada grupo 01 folha de papel 40Kg, EVA, pincéis atômicos, cola, tesoura e régua. $\mathrm{O}$ tempo delimitado para a realização foi de 40 minutos. Após, cada grupo procedeu à apresentação de seu cartaz, mostrando como seria realizada a Feira Educacional em sua escola.

TARDE - Gravidez na Adolescência. Aula Expositiva-interativa: buscou-se apresentar conceitos de alguns autores para fundamentar as discussões sobre a adolescência, puberdade e estatísticas nacionais e internacionais sobre o quantitativo de adolescentes grávidas e a relevância deste tema. Dinâmica de Grupo - Batata Quente: consistiu em formar um círculo composto por todos os participantes. Ao som de uma música animada, uma bola circulava entre os participantes, de mão em mão; quando a música paralisava o participante que ficou com a bola (batata quente) na mão tinha que citar um método contraceptivo; aqueles que não soubessem ou repetissem um método já citado teriam que pagar uma "prenda". Atividade Prática - Mesa redonda: primeiramente foi realizada leitura de um texto reflexivo sobre o assunto. Depois, deu-se início à mesa redonda; ao centro da sala tinha um recipiente contendo perguntas voltadas às questões familiares e sociais da gravidez na adolescência. Aleatoriamente, os participantes retiraram as perguntadas e as respondiam, expondo suas opiniões sobre a problemática explicitada.

Os temas discutidos nestes 02 dias permitiram a reflexão de como esta temática é tratada em sala de aula na realidade de cada professor, restringindo-se basicamente às disciplinas de ciências e biologia, o que inviabiliza a discussão e a problematização de alguns aspectos centrais em relação às questões de corpos, gêneros e sexualidades ${ }^{8}$.

\section{Dia: 15/09/2010}

MANHÃ - Abuso e Violência Sexual. Aula Expositiva-interativa: explicaram-se os conceitos, os principais sinais de cada tipo de abuso e o tratamento necessário. Dinâmica de Grupo - Como lidar com o abuso sexual?: consistiu em dividir a sala em 04 grupos. Cada grupo recebeu uma faixa etária e estabeleceu metodologias para lidar com o aluno em cada idade, discutindo exemplos de trabalhos que já são e/ou podem ser desenvolvidos nas escolas para identificar alunos com problemas relacionados a abuso e violência sexuais Atividade Prática - Teatro livre: a turma foi dividida em 02 grupos. Com o tema envolvendo "abuso e violência sexual", eles montaram um teatro com duração máxima de 10 minutos de apresentação. Para tal, criaram uma história fictícia ou contaram algum caso real já vivenciado.

$T A R D E$ - Aborto - interrupção da gravidez. Aula Expositiva-interativa: iniciou-se com um debate sobre quando a vida realmente se inicia: no momento em que o espermatozóide fecunda o óvulo ou mais tarde? Para fundamentar opiniões, foram apresentadas algumas linhas de pensamento que defendem o início da vida em diferentes momentos. Também abordaram-se questões referentes ao conceito científico de abortamento, sua incidência, classificação, o aborto e código penal de 1940, a proposta de descriminalização do aborto e os riscos secundários à prática do abortamento clandestino. Foram evidenciadas questões sobre a educação sexual, a informação e a prevenção como armas para a redução da incidência de casos de aborto ilegal e de mortes materno-infantis. Dinâmica de Grupo - Discutindo casos: objetivou-se mostrar possíveis casos em que o aborto poderia ser cogitado ou praticado, a fim de extrair dos participantes qual seria sua postura caso estivessem passando por tal situação. A turma foi dividida em 04 grupos e para cada um deles foi distribuído um caso diferente. Ao final, cada grupo expôs sua situação e apresentou sua opinião de desfecho da história. Atividade Prática - Exercício de reflexão sobre 
sentimentos: o objetivo foi encorajar uma reflexão sobre os sentimentos de uma pessoa que se descobre grávida indesejavelmente e opta pela realização de um aborto. Sua realização durou em média 01 hora e 30 minutos. Foram dados 04 pedaços de papéis a cada participante e solicitado que escrevessem em cada papel o seguinte: 1- Nome de uma parte importante de seu corpo; 2- Nome da pessoa mais importante de sua vida; 3-Um sentimento importante; 4-Um bem material mais importante que possui. Logo após, foi proporcionado um ambiente favorável ao relaxamento e reflexão e solicitado à turma para que se imaginassem em uma situação difícil, que deveriam escolher um dos valores descritos para descartar em um lixo presente no meio do círculo formado. O descarte de valores ocorreu 03 vezes, até que cada participante ficasse apenas com 01 deles. Ao final, cada um expôs suas dificuldades em ter que se desfazer de alguns valores.

Dia: 16/09/2010

$M A N H \tilde{A}$ - DSTs. Aula Expositiva-interativa: abordaram-se a conceituação, a gravidade, as consequências, o tratamento e a prevenção das DSTs, as quais foram distribuídas didaticamente em doenças que causam verrugas, doenças que causam feridas e doenças que causam corrimentos. Dinâmica de Grupo - Contatos pessoais: O objetivo foi demonstrar a facilidade de transmissão sexual das DSTs, entregando aos participantes 01 folha com apenas 01 figura já desenhada pelo facilitador. Como participaram 20 pessoas, os desenhos ficaram distribuídos da seguinte forma: 04 triângulos; 06 quadrados; 10 círculos. Os participantes dançaram pela sala e conversaram com seus colegas, com a finalidade de manter integração. Quando a música parou, os participantes desenharam em seu papel a figura da pessoa mais próxima, sendo que esse processo se repetiu 05 vezes. Após, foi esclarecido à turma o significado de cada figura: círculo - pessoa sadia, quadrado - portador de DST e triângulo - portador de HIV. Assim, foi promovida uma discussão sobre a facilidade de relacionamento entre as pessoas e a possível chance de se adquirir alguma DST. Atividade Prática - Gincana (Passa ou Repassa): A turma foi dividida em 02 grupos com 10 participantes em cada. A dinâmica consistiu em um jogo de perguntas, com pontuação variando de 01 a 05 pontos, colocadas em um recipiente para serem retiradas pelos participantes. Venceu o grupo com maior somatória nos pontos.

TARDE - HIV e AIDS. Aula Expositiva-interativa: iniciou-se com apresentação de dados apontados pelo Ministério da Saúde acerca da incidência crescente da contaminação HIV / AIDS entre os jovens brasileiros. Pontuaram-se questões como os vários fatores que podem tornar os adolescentes vulneráveis ao HIV / AIDS; fisiopatologia da doença; formas de transmissão; comportamentos sexuais de risco; diferença entre portador do vírus HIV e AIDS; classificação clínica; tratamento; prevenção e ênfase no preconceito $x$ respeito. Após, lançamos a seguinte pergunta aos participantes: Como lidar com essa situação e qual o seu papel como agente transformador dessa realidade? Para responder a esse questionamento, apresentamos algumas sugestões aos participantes de como agir em determinadas situações e como tornar a abordagem desse assunto uma realidade no cotidiano escolar de seus alunos. Dinâmica de Grupo - A canoa virou: objetivou-se discutir a natureza do preconceito e maneiras pelas quais discriminamos ou não alguém. A duração foi em média de 20 minutos. Foram formadas 05 equipes e apresentada a seguinte situação: "Cada grupo está em um barco em alto-mar. O barco bate em um recife e pode afundar a qualquer momento. Vem um barco salva-vidas que tem capacidade de transportar todas as pessoas menos uma. Por isso, cada grupo vai excluir um membro, baseado em critérios decididos e aceitos pelo grupo". Após, os excluídos foram chamados para frente e apresentaram os motivos pelos quais o grupo teve essa decisão e relataram qual o sentimento de exclusão. Os não excluídos também relataram o que sentiram ao terem de definir os critérios de exclusão. Atividade Prática - Oficina (montagem de painéis): foram formados 03 grupos e distribuídos materiais para a confecção dos painéis: papel 40Kg, EVA, 
papel cartão, barbante, pincéis atômicos, cola, tesoura e régua. Assim, foi solicitado a cada grupo que representassem, através de desenhos, seus sentimentos em relação ao problema tratado nesse período, não sendo permitido utilizar a forma escrita. O tempo para a elaboração foi de 40 minutos; logo após, os grupos trocaram de painéis e os interpretaram.

\section{Dia: $17 / 09 / 2017$}

MANHÃ - Uso de drogas e violência. Aula Expositiva-interativa: foi mostrada a diferença entre drogas lícitas e ilícitas, enfatizando sua ação negativa. Atividade Prática - Teatro livre: divididos em 02 grupos, eles deveriam montar uma peça teatral envolvendo o tema "violência e uso de drogas", que seria apresentada no máximo em 10 minutos. Para desenvolver a tarefa, poderiam basear-se em histórias reais ou criar alguma situação.

TARDE - Prevenção à Saúde. Neste período do último dia de capacitação, os facilitadores trataram de temas como Saúde e Qualidade de Vida, Meio ambiente e saúde e Planejamento Familiar. Após as abordagens, foi realizado um coquetel de encerramento como forma de confraternização entre a equipe.

A temática abordada nos dias 15,16 e 17 permitiu a reflexão por parte dos professores sobre a importância de projetos destinados à prevenção ao uso de drogas e das DSTs, nos quais pudessem enfatizar a relação entre esses temas, capacitar docentes e expandir o conhecimento dos discentes sobre o assunto. A escola, devido à possibilidade de acesso aos jovens e à natureza educacional do seu trabalho, é considerada, em todo o mundo, o locus privilegiado dos programas de prevenção dirigidos aos adolescentes ${ }^{9}$. Porém, como relatado por alguns participantes do projeto e pela literatura científica, observam-se a relutância e o despreparo das escolas para lidar com os problemas sociais da sociedade atual, especialmente com temas considerados tabus 9 .

\section{Resultados e impactos}

Com a realização deste projeto, através dos relatos de experiências verbalizados, foi possível evidenciar a limitada e esporádica abordagem dos Temas Transversais no cotidiano escolar dos professores participantes, sendo a temática tratada de forma isolada e desconexa aos conteúdos trabalhados em sala de aula.

Assim, a capacitação favoreceu aos professores o conhecimento de ferramentas metodológicas para a abordagem integrada dos Temas Transversais às disciplinas escolares, contribuindo para uma nova prática pedagógica interdisciplinar, pois "[...]a interdisciplinaridade envolve perceber que um conhecimento mantém um diálogo constante com outros conhecimentos, pois não estão fragmentados"'2 (p. 04).

Além disso, foi possível contribuir para a qualificação e valorização da formação continuada de professores da educação básica municipal, por meio da articulação Universidade-Escola e da implementação de projetos de extensão.

A formação continuada, realizada através de cursos, encontros e palestras promovidos pelas Secretarias de Educação ou por uma rede de escolas, por exemplo, é a garantia do desenvolvimento profissional permanente, feita por meio do estudo, da reflexão, da discussão e da confrontação das experiências docentes ${ }^{6}$. Para tanto, é necessário que os professores tenham um tempo fora da sala de aula em contextos nos quais se sintam bem para falar sobre o seu trabalho, refletir a respeito de sua prática pedagógica, sistematizar as metodologias usadas, compartilhar com os colegas os problemas enfrentados e fazer planejamentos coletivos ${ }^{6}$, sendo exatamente o que foi executado com esse projeto.

Durante a execução algumas dificuldades foram enfrentadas, como o entrosamento entre a equipe e o gerenciamento do tempo das dinâmicas e práticas. Porém, com o 
decorrer das atividades, foi estabelecida uma relação de confiança entre facilitadores e participantes e melhor gerenciamento do tempo de socialização das tarefas.

Ao término das atividades foi solicitada uma avaliação do curso à turma através do preenchimento de uma ficha elaborada pelos autores, contendo 06 itens a serem avaliados (recursos didáticos; conteúdo abordado; técnicas utilizadas nas dinâmicas e práticas; atividades sugeridas na parte prática; segurança dos ministrantes e entrosamento da turma com os ministrantes) e 04 opções de julgamento (excelente; bom; regular e ruim). Os resultados apontaram para as classificações "bom" e "excelente".

O forte impacto no município pela realização e repercussão da importância dos conteúdos abordados neste curso propiciaram novas oportunidades aos elaboradores, por meio de convites para participar de outros eventos institucionais e aplicar o projeto em municípios vizinhos, ações que fortaleceram a integração da UEPA com as Secretarias de Educação e Saúde de Tucuruí e região.

\section{Considerações finais}

A formação continuada deve ser um processo contínuo, permanente e integrado ao cotidiano dos professores e da instituição escolar. O professor precisa estar em constante processo de aprendizagem, avaliando e refazendo o seu trabalho com leituras, pesquisas e trocas de experiências ${ }^{6}$.

Este projeto permitiu concluir que para que a transversalidade e interdisciplinaridade sejam efetivadas não basta apenas a inserção de Temas Transversais nas disciplinas escolares, sendo estes tratados isoladamente por cada professor. Torna-se imprescindível que as relações entre comunidade acadêmica e escolar sejam fomentadas por meio da implementação de projetos de pesquisa e de extensão que estimulem o planejamento e desenvolvimento de ações para a promoção da saúde de forma permanente nas escolas. Entendeu-se que a "[...] capacitação de professores para ensinar e aprender promoção e educação em saúde deve ser permanente, necessariamente ligada a uma ação dinâmica, ininterrupta e atualizada" ${ }^{5}$ (p. 100).

Com os resultados positivos alcançados, sugere-se a expansão de projetos deste tipo para serem aplicados em outras escolas do município e região. Os professores perceberam também a importância de seu papel como educadores para a promoção da saúde na escola.

\section{Referências}

1. Brasil. Ministério da Educação. Secretaria da Educação Fundamental. Parâmetros curriculares nacionais: apresentação dos temas transversais e ética. 3. ed. Brasília: Ministério da Educação; 2001. (vol. 8).

2. Almeida TJB. Abordagem dos temas transversais nas aulas de ciências do ensino fundamental, no distrito de Arembepe, município de Camaçari-BA. Candombá. 2006;2(1):1-13 [citado em 2017 Ago 22]. Disponível em: http://revistas.unijorge. edu.br/candomba/2006-v2n1/pdfs/TeresaAlmeida2006v2n1.pdf

3. Demo P. Desafios modernos da educação. 10. ed. Petrópolis: Vozes; 2000.

4. Menezes ET, Santos TH. Verbete temas transversais. In: Educabrasil. Dicionário interativo da educação brasileira. São Paulo: Midiamix; 2001 [citado em 2017 Ago 15]. Disponível em: http://www.educabrasil.com.br/temas-transversais/

5. Iervolino SA, Pelicioni MCF. Capacitação de professores para a promoção e educação em saúde na escola: relato de uma experiência. Rev Bras Cresc Desenv Hum. 2005;15(2):99-110. http://dx.doi.org/10.7322/jhgd.19762.

6. Verdinelli MM. Formação continuada de professores do ensino fundamental subsidiada pela pedagogia histórico-crítica e teoria histórico-cultural [dissertação]. Maringá: Universidade Estadual de Maringá; 2007 [citado em 2018 Fev 3]. Disponível em: http://www.ppe.uem.br/dissertacoes/2007\%20-\%20Marilsa_Maria_Verdinelli.pdf

7. Gallo S. Transversalidade e educação: pensando uma educação não-disciplinar. In: Alves N, Garcia RL, editores. O sentido da escola [Internet]. Rio de Janeiro: DP\&A; 2000. p. 17-41 [citado em 2017 Ago 15]. Disponível em: http://www.lite. fe.unicamp.br/papet/2003/ep403/transversalidade_e_educacao.htm

8. Barros SC, Ribeiro PRC. Educação para sexualidade: uma questão transversal ou disciplinar no currículo escolar? REEC. 2012;11(1):164-87 [citado em 2017 Ago 25]. Disponível em: http://reec.uvigo.es/volumenes/volumen11/REEC_11_1_9_ex570. pdf 
9. Soares CB, Jacobi PR. Adolescentes, drogas e aids: avaliação de um programa de prevenção escolar. Cadernos Pesquisa. 2000;(109):213-37. http://dx.doi.org/10.1590/S0100-15742000000100010.

\section{Contribuição dos autores}

Todos os autores participaram desde a concepção e desenvolvimento do estudo, coleta e levantamento da literatura utilizada, realizaram a redação e revisão crítica. 